\title{
Stent-assisted Coil Embolization to Treat Extracranial Carotid Artery Aneurysm 13 Years after Endarterectomy: A Case Report
}

\author{
Jun Haruma, ${ }^{1}$ Nobuyuki Hirotsune, ${ }^{2}$ Tetsuo Oka, ${ }^{1}$ and Tadashi Arisawa ${ }^{1}$
}

\begin{abstract}
Development of extracranial carotid artery aneurysm (ECCA) after carotid endarterectomy (CEA) is a rare complication, occurring in connection with $<1 \%$ of all CEAs. The main causes are infection, suture failure, and degeneration of arterial wall or patch. The traditional treatment has been operative repair, which can present a significant technical challenge owing to reoperative neck inflammation and potential cranial nerve injuries. Here, we report a case of successful stent-assisted coil embolization for right noninfectious ECCA. A 63-year-old female was admitted to our hospital for a 3-cm pulsating mass in her right midneck. Doppler examination and digital subtraction angiography revealed a large $(15 \mathrm{~mm})$ ECCA at the right common carotid artery (CCA). Thirteen years earlier, eversion CEA with patch angioplasty and abbreviation of the internal carotid artery (ICA) had been performed for a symptomatic $80 \%$ diameter stenosis with transient ischemic attack at another hospital. As the patient refused blood transfusion for religious reasons, we treated her with stent-assisted coil embolization, which achieved nearly complete obliteration of the aneurysm while preserving the parent artery patency. Three months after initial treatment, ultrasound revealed complete occlusion of the ECCA, but also showed stent shortening. Hence, we performed an additional stent placement so as to overlap the previous stent by $2.5 \mathrm{~cm}$. Six months after initial treatment, carotid duplex ultrasound confirmed a good outcome. This procedure is an excellent choice for highrisk patients, and a larger case series is needed to establish this technique as the treatment of choice for ECCAs.
\end{abstract}

Keywords: extracranial carotid artery aneurysm after carotid endarterectomy, endovascular treatment, stentassisted coil embolization

\section{Introduction}

Development of extracranial carotid artery aneurysm (ECCA) after carotid endarterectomy (CEA) is a rare complication, occurring in connection with $<1 \%$ of all CEAs. ${ }^{1)}$ ECCA can occur after a long postoperative interval, even

${ }^{1}$ Department of Neurosurgery, Fukuyama City Hospital, Fukuyama, Hiroshima, Japan

${ }^{2}$ Department of Neurosurgery, Hiroshima City Hospital, Hiroshima, Hiroshima, Japan

Received: February 14, 2019; Accepted: June 6, 2019

Online December 18, 2019

Copyright $(2020$ by The Japan Neurosurgical Society This work is licensed under a Creative Commons AttributionNonCommercial-NoDerivatives International License. years after the CEA. ${ }^{2)}$ Several surgical treatments have been described, and an endovascular approach including the insertion of a covered or bare metal stent is an alternative option. ${ }^{3)}$

Here, we report on a clinical case of ECCA occurring 13 years after eversion CEA by the endovascular approach, which we successfully obliterated using the stent-assisted coil embolization technique.

\section{Case Report}

A 63-year-old female was admitted to our hospital for a $3-\mathrm{cm}$ pulsating mass in her right midneck that had gradually increased in size over the preceding 6 months. Doppler examination confirmed that the mass was an ECCA in the right common carotid artery (CCA). Laboratory data concerning the peripheral blood revealed no anomalies in the white cells count and in the indices of inflammation. The patient had a history of CEA with patch angioplasty by Dacron at another hospital 13 years previously. The CEA had been performed for a symptomatic $80 \%$ diameter stenosis with transient ischemic attack (TIA). She had tolerated the procedure well and had been discharged to her home on postoperative day 14. Routine follow-up at 2 years had shown the patient to be doing well with no new TIA or strokes. Physical examination at that time had also noted no abnormalities of the incision site or at the patient's neck. Additionally, computed tomography angiography 2 years after CEA did not show restenosis or aneurysm formation (not shown).

At the present admission, digital subtraction angiography revealed a wide and irregular ECCA formation originating in the right CCA and measuring $15 \times 13 \mathrm{~mm}$. The largest diameter of the patient's CCA was $10 \mathrm{~mm}$, whereas the normal vessel diameter is $6 \mathrm{~mm}$. Magnetic resonance imaging (MRI) showed that the ECCA was partially thrombosed (Fig. 1). Because the patient refused blood transfusion and requested bloodless surgery for religious reasons, coil embolization and carotid artery stenting device tunneled within a carotid stent implantation across the pseudoaneurysm was considered after a detailed discussion with the patient. Pre-procedural clopidogrel $75 \mathrm{mg}$ and aspirin $100 \mathrm{mg}$ daily for 7 days were commenced.

We planned to use proximal and distal balloon protection in preparation for rupture. Full heparinization was instituted before the procedure. Under local anesthesia with a right brachial artery and femoral artery approach, a 4-F Simonds catheter and 8-F FlowGate (Stryker Neurovascular, Fremont, CA, USA) were placed in the right CCA as follows. First, an Excelsior SL-10 microcatheter (Stryker Neurovascular, Fremont, CA, USA) was placed into the aneurysm from the brachial artery. Then, a Wall $10 \times 31$-mm carotid stent (Boston Scientific, Natick, MA, USA) 

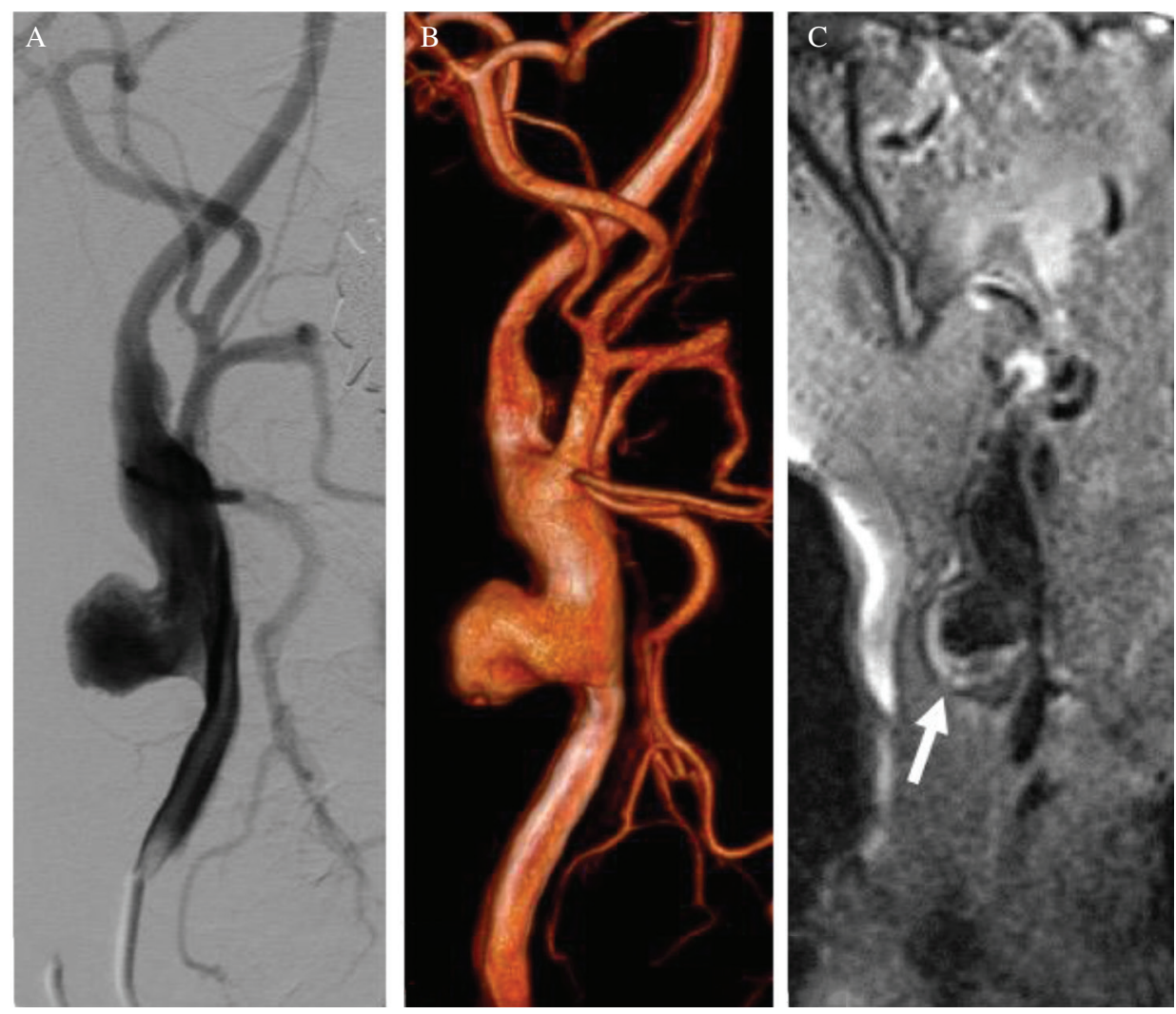

Fig. 1 (A) Common carotid artery (CCA) angiogram revealed the patient's pseudoaneurysm (PA). (B) Threedimensional digital subtraction angiography confirmed a PA $(15 \times 13 \mathrm{~mm})$ originating in the right carotid artery. The largest vessel diameter of the CCA was $10 \mathrm{~mm}$ whereas the normal vessel diameter is $6 \mathrm{~mm}$. (C) Magnetic resonance imaging - sampling perfection with application optimized contrast using different flip angle evolution showed that the extracranial carotid artery aneurysm was partially thrombosed (arrow). was deployed in combination with proximal and distal balloon protection (Carotid Guardwire PS; Medtronic, Minneapolis, MN, USA). Another microcatheter (Headway 17; MicroVention, Tustin, CA, USA) was placed in the aneurysm. The aneurysm was occluded using 25 detachable coils according to the double catheter technique. Finally, because the expansion of the proximal stent edge was not sufficient, we performed balloon angioplasty with a $4.5-\mathrm{mm}$ diameter balloon in combination as described above (Fig. 2).

Postoperative recovery was uneventful, and the patient was discharged 5 days later with instructions for continued combined aspirin and clopidogrel therapy. Her neck swelling and pulsation of the mass disappeared 14 days later.

Ultrasound scans 3 months after the initial treatment revealed complete occlusion of the pseudoaneurysm, but also showed stent shortening toward the distal end and stenosis at the proximal portion of the stent. Out of consideration for the possibility of recanalization, we decided to perform retreatment. Under local anesthesia, a guiding catheter (ASAHI FUBUKI Dilator; Asahi-Intec, Aichi, Japan) was introduced into the right CCA via a femoral route. A Wall $10 \times 24-\mathrm{mm}$ carotid stent was deployed so that it overlapped with the first device by about $2.5 \mathrm{~cm}$ with proximal and distal protection balloon protection devices as before. Finally, post-balloon angioplasty was performed with a 7.0-mm diameter balloon (Fig. 3). The patient was discharged 4 days later with instructions to continue combined aspirin and clopidogrel therapy for 1 month and aspirin monotherapy thereafter for 3 months. Carotid duplex ultrasound scans at 1 and 6 months confirmed a desirable outcome (not shown).

\section{Discussion}

Post-CEA ECCA is a rare complication and may occur even after a long postoperative interval. In our case, it was initially thought that suture degeneration had occurred for some reason, but the true immediate cause remains unknown. No gold-standard treatment for ECCA following CEA has been established to date, though the stent-assisted coil embolization technique is considered the most minimally invasive and effective treatment method for ECCA.

According to the available literature, carotid artery ECCA following CEA occurs in approximately $0.3-0.6 \%$ of cases. ${ }^{1)}$ The pathophysiology of ECCA formation has been reported to include suture degeneration, infection, clamp-induced damage, and shunt use. According to one report, ECCAs are twice as common after patch angioplasty as they are after primary closure. ${ }^{4)}$ Another report, however, has reported a $0.33 \%$ incidence of ECCA after patch angioplasty versus a $0.25 \%$ incidence following primary closure, which difference does not reach statistical significance. The etiology of ECCA can be difficult to define. Histologic findings are required to precisely define the pathologic characteristics of ECCA in the absence of a clear cause. Idiopathic ECCA has most frequently been reported to be related to arterialization. According to one report, however, ECCA after CEA showed dilation of venous patches without evidence of arterialization. ${ }^{5)}$ Thus, ECCA has different histological origins depending on whether it is idiopathic or occurring after CEA.

Extracranial carotid artery aneurysm after CEA has a broad temporal distribution of occurrence, developing at any time from days to many years after surgery. ${ }^{1)}$ Usually, however, it is 

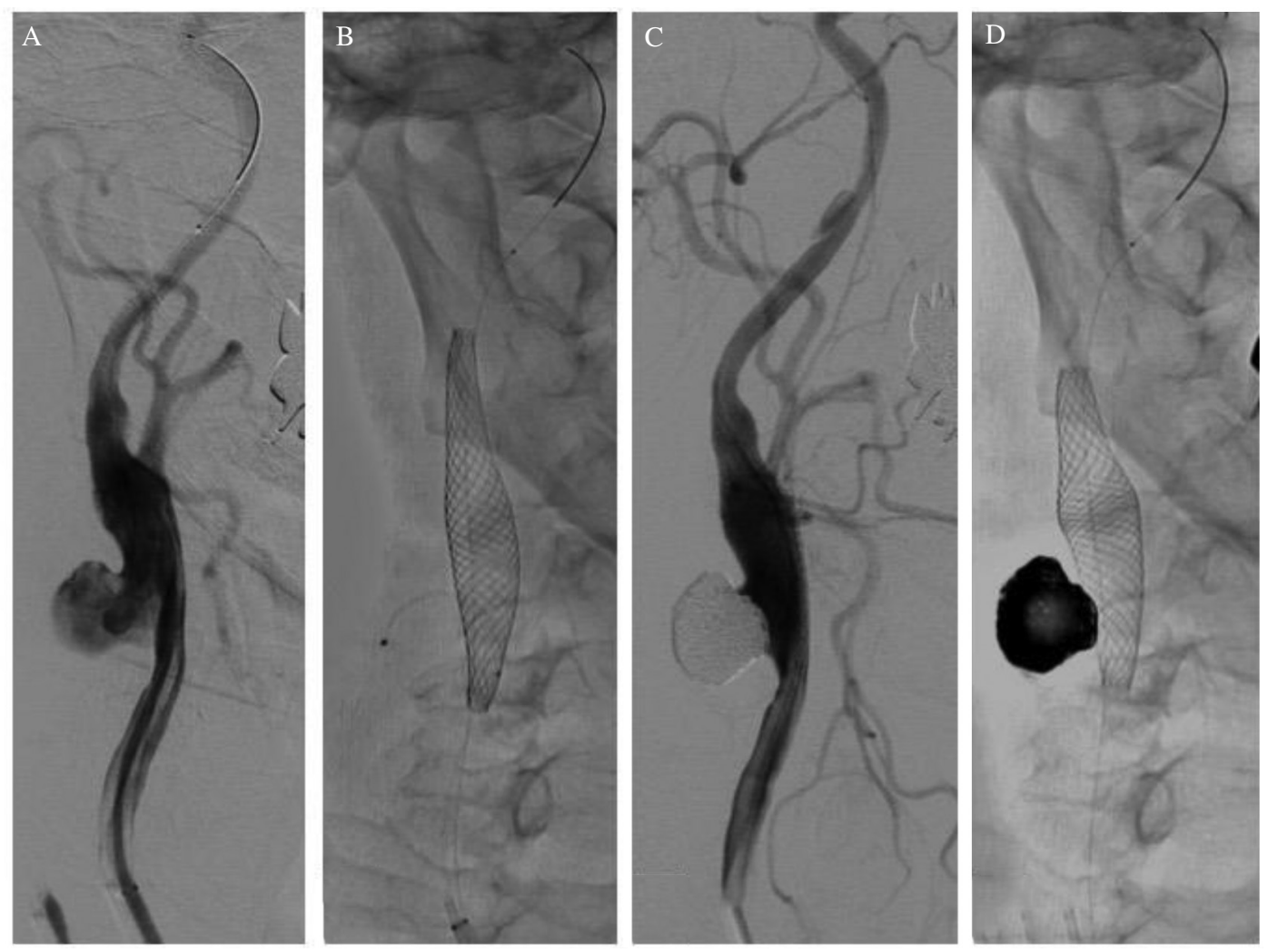

Fig. 2 (A) Common carotid artery angiogram (CCAG) before stentassisted coil embolization (SAC) for the right extracranial carotid artery aneurysm (ECCA). (B) The Wall $10 \times 31-\mathrm{mm}$ carotid stent was placed successfully from the right internal carotid artery to the CCA to cover the aneurysm neck. (C and D) The CCAG after SAC showing coil occlusion of the ECCA and patency of the parent arteries.
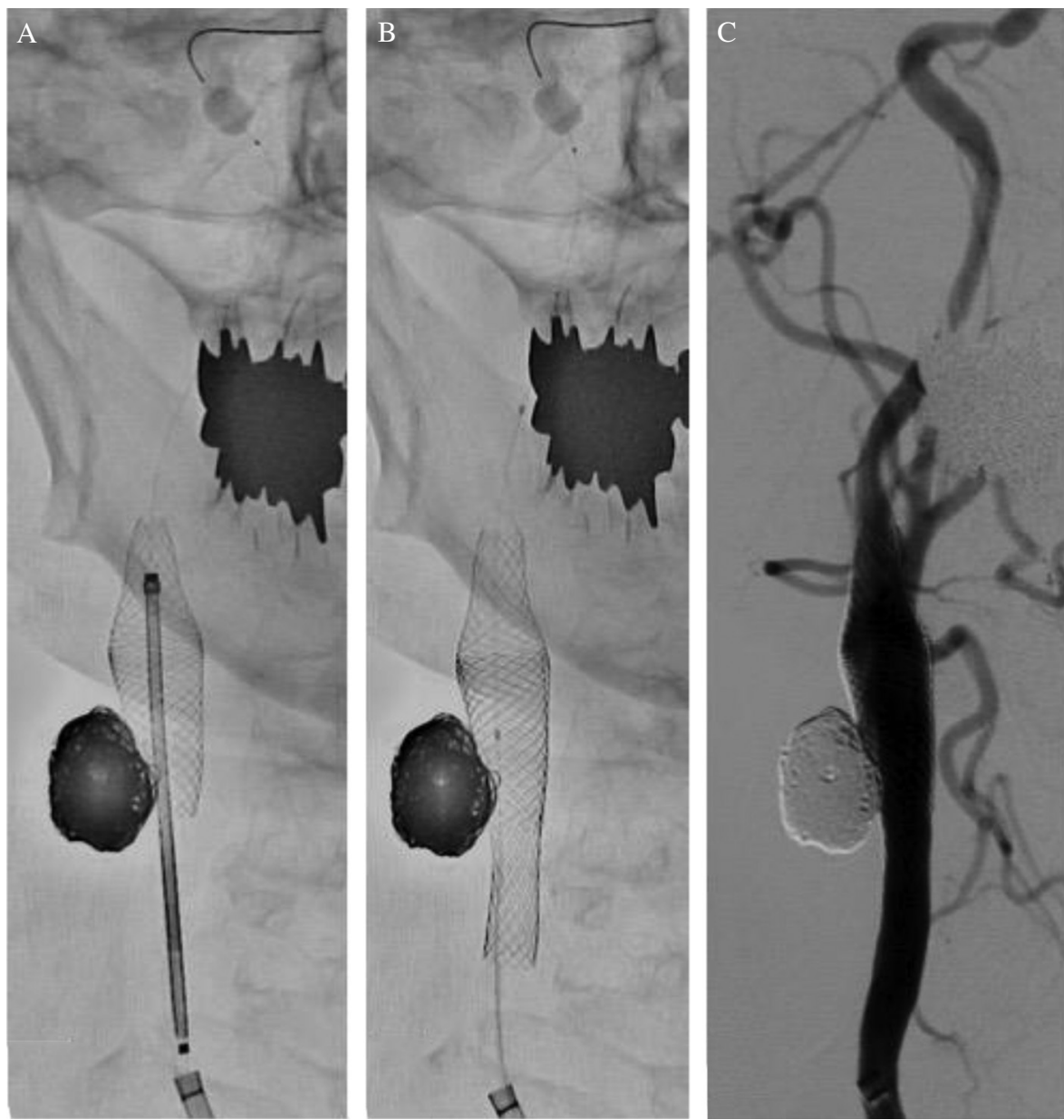

Fig. 3 (A and B) The first Wall stent was shortened to the center of the neck of the aneurysm. The Wall $10 \times 24-\mathrm{mm}$ carotid stent was placed from the carotid bulb. The second stent was placed to overlap the first stent by $2.5 \mathrm{~cm}(\mathrm{C})$ common carotid artery angiogram after treatment. Recanalization of the pseudoaneurysm was not observed. 
Table 1 Previous case reports of extracranial carotid artery aneurysm occurring more than 10 years after CEA

\begin{tabular}{|c|c|c|c|c|c|c|c|c|}
\hline Reference & Year & Age & Sex & Interval (Years) & CEA closure & Infection & Presenting symptoms & Treatment \\
\hline McCollum et al. ${ }^{6}$ & 1979 & $\mathrm{~N} / \mathrm{K}$ & $\mathrm{N} / \mathrm{K}$ & 15 & Dacron patch & Yes & Painful neck mass & Surgery repair (Ligation) \\
\hline Borazjani et al. ${ }^{7)}$ & 2003 & 76 & $\mathrm{~F}$ & 10 & Dacron patch & Yes & Painful neck mass & $\begin{array}{l}\text { Surgery repair (polytetrafluoro- } \\
\text { ethylene interposition graft) }\end{array}$ \\
\hline Martin et al. ${ }^{2)}$ & 2005 & 68 & M & 17 & $\begin{array}{l}\text { Saphenous } \\
\text { vein patch }\end{array}$ & No & $\begin{array}{l}\text { Asymptomatic neck } \\
\text { mass }\end{array}$ & Endovascular (Covered stenting) \\
\hline Litwinski et al. ${ }^{8)}$ & 2006 & 75 & $\mathrm{~F}$ & 10 & Patch closure & Yes & Painful neck mass & Surgery repair (Vein graft) \\
\hline This report & & 63 & $\mathrm{~F}$ & 13 & Dacron patch & No & $\begin{array}{l}\text { Asymptomatic neck } \\
\text { mass }\end{array}$ & $\begin{array}{l}\text { Endovascular } \\
\text { (Stent with coil embolization) }\end{array}$ \\
\hline
\end{tabular}

N/K: not known.

more common within the first several months after surgery. As far as we can ascertain, there are four previous reports of ECCA occurring more than 10 years after CEA., ${ }^{2,-8)}$ Three of these were infectious aneurysms. Including the present case, therefore, there are only two recorded instances of noninfectious ECCA occurring more than 10 years after CEA (Table 1). Once a diagnosis is made, treatment is recommended to avoid rupture, distal embolization, nervous compression and/or the emergence of neurologic deficits arising from thromboembolic events; when ECCAs are left untreated, several studies have reported a stroke prevalence of 50\% and a mortality of $60-70 \%$.9)

Endovascular treatment according to the stent-assisted coil embolization technique has been reported as an effective treatment for intracranial aneurysm, ${ }^{10)}$ and open repair with autogenous arterial reconstruction is the current gold standard for the treatment of ECCA even if it is post CEA, but a treatment regime intended specifically for the management of ECCA following CEA has not been established. ${ }^{11,12)}$ Several therapeutic strategies are available, including medical therapy as well as direct surgical treatment, which may require sacrifice of the parent vessel or artery reconstruction with bypass grafting. However, the surgical management of ECCA can be challenging, especially if there is a difficulty with surgical exposure. ${ }^{13)}$ In a review on open operative repairs of carotid aneurysms, El-Sabrout and Cooley ${ }^{14)}$ found a major stroke and death rate of 9\% (range, 3-20\%), compared with $21 \%$ in patients who did not undergo surgical repair. Alongside open surgical repair, endovascular techniques with stents and stent grafts are increasingly being used. Types of stents reported in the literature include covered stents, bare metal stents, and Flow-Diverter stents. ${ }^{15-17)}$ In situations where the vascular endothelium has failed, such as cases with dissection or a failed CEA, the endovascular approach with a metal stent is useful. ${ }^{18)}$

In this case, the continuity between the aneurysm and the common carotid artery was confirmed adventitia by MRI (Fig. 1). Therefore, this case is considered to be a true aneurysm wherein the vein patch has expanded. We used a bare metal stent plus coil embolization instead of a covered stent to exclude the ECCA. In the present case, a covered stent could not be used because the difference in the vessel diameter of the CCA was large. ${ }^{15)}$ Likewise, a Flow-Diverter stent could not be used because it is not available in the size that would have been needed for this case. ${ }^{17)}$ In previous reports, overlapping bare metal stents have had good short-term results, but no long-term results have been reported until now. ${ }^{19)}$ Therefore, we decided that it was necessary to use a coil combination. Furthermore, the combination of stent plus coil embolization was expected to stabilize the coil placement in the enlarged portion while preserving the parent blood vessel, and also to promote re-endothelialization. ${ }^{10,20)}$ One disadvantage of intravascular treatment compared to surgical operation is that the hemostasis technique cannot be performed at the time of rupture. Particularly in this case, as this patient refused blood transfusion, preparation for a possible rupture was necessary. Hence, we used a proximal and a distal balloon in preparation for possible rupture. In addition, we chose the double catheter method so that coil insertion could be performed just in case. There is room for discussion regarding how much coil should be used. In this case, the cervical swelling disappeared after treatment even though coil was used. We had expected some shortening of the stent, but we did not administer any additional treatment to prevent it because the stent was covering the lesion well at the time of the operation. ${ }^{21)}$ We expect that, in future cases, a two-stent technique could be used to prevent such recurrence. Several studies have shown that restriction of the aneurysmal flow is more complete if the ratio of the effective mesh area to the total surface of the stent is small, regardless of the aneurysm size. ${ }^{22)}$ Complete obliteration of the aneurysm as well as perfect preservation of the carotid patency were achieved. For antiplatelet therapy, because the risk of thrombosis was considered high, ${ }^{23)}$ we chose dual antiplatelet therapy as reported previously. ${ }^{3)}$

However, careful follow-up observation is necessary, because the safety and efficacy of this method in the long term is still unconfirmed.

\section{Conclusion}

Post-CEA ECCA is a rare complication that may occur even after a long postoperative interval. Our experience has shown that the combination of stent plus coil embolization is a successful and viable option in patients with ECCA after CEA. We believe that this is less invasive and safer than open repair in patients without signs of infection. This makes 
it an excellent choice for high-risk patients. A larger case series is needed to establish this technique as the treatment of choice for carotid ECCAs.

\section{Conflicts of Interest Disclosure}

All authors have no conflicts of interest.

\section{References}

1) Abdelhamid MF, Wall ML, Vohra RK: Carotid artery pseudoaneurysm after carotid endarterectomy: case series and a review of the literature. Vasc Endovascular Surg 43: 571-577, 2009

2) Martin ND, Carabasi RA, Bonn J, Lombardi J, DiMuzio P: Endovascular repair of carotid artery aneurysms following carotid endarterectomy. Ann Vasc Surg 19: 913-916, 2005

3) Seward CJ, Dumont TM, Levy EI: Endovascular therapy of extracranial carotid artery pseudoaneurysms: case series and literature review. J Neurointerv Surg 7: 682-689, 2015

4) Ehrenfeld WK, Hays RJ: False aneurysm after carotid endarterectomy. Arch Surg 104: 288-291, 1972

5) Yamamoto Y, Piepgras DG, Marsh WR, Meyer FB: Complications resulting from saphenous vein patch graft after carotid endarterectomy. Neurosurgery 39: 670-675; discussion 675-676, 1996

6) McCollum CH, Wheeler WG, Noon GP, DeBakey ME: Aneurysms of the extracranial carotid artery. Twenty-one years' experience. Am J Surg 137: 196-200, 1979

7) Borazjani BH, Wilson SE, Fujitani RM, Gordon I, Mueller M, Williams RA: Postoperative complications of carotid patching: pseudoaneurysm and infection. Ann Vasc Surg 17: 156-161, 2003

8) Litwinski RA, Wright K, Pons P: Pseudoaneurysm formation following carotid endarterectomy: two case reports and a literature review. Ann Vasc Surg 20: 678-680, 2006

9) Hertz JA, Minion DJ, Quick RC, Moore EM, Schwartz TH, Endean ED: Endovascular exclusion of a postendarterectomy carotid pseudoaneurysm. Ann Vasc Surg 17: 558-561, 2003

10) Lopes D, Sani S: Histological postmortem study of an internal carotid artery aneurysm treated with the Neuroform stent. Neurosurgery 56: E416, 2005

11) Fankhauser GT, Stone WM, Fowl RJ, et al.: Surgical and medical management of extracranial carotid artery aneurysms. J Vasc Surg 61: 389-393, 2015
12) Welleweerd JC, den Ruijter HM, Nelissen BG, et al.: Management of extracranial carotid artery aneurysm. Eur J Vasc Endovasc Surg 50: $141-147,2015$

13) Kubaska SM, Greenberg RK, Clair D, et al.: Internal carotid artery pseudoaneurysms: treatment with the Wallgraft endoprosthesis. J Endovasc Ther 10: 182-189, 2003

14) El-Sabrout R, Cooley DA: Extracranial carotid artery aneurysms: Texas Heart Institute experience. J Vasc Surg 31: 702-712, 2000

15) Ergun O, Celtikci P, Canyigit M, Birgi E, Hidiroglu M, Hekimoglu B: Covered stent-graft treatment of a postoperative common carotid artery pseudoaneurysm. Pol J Radiol 79: 333-336, 2014

16) Troutman DA, Mohan CR, Samhouri FA, Sohn RL: Endovascular repair of carotid artery pseudoaneurysm after carotid endarterectomy with self-expanding covered stents-a long-term follow-up. Ann Vasc Surg 24: 954.e13-954.e16, 2010

17) Young M, Imbarrato G, Gordhan A: Symptomatic post endarterectomy common carotid artery pseudoaneurysm treated with combination of flow diverter implantation and carotid stenting. Neurointervention 13: 54-57, 2018

18) Terramani TT, Workman MJ, Loberman Z, et al.: Adjunctive endovascular techniques in the management of postoperative carotid artery pseudoaneurysms - useful armamentarium for vascular surgeonsthree case reports. Vasc Endovascular Surg 37: 207-212, 2003

19) Varetto G, Castagno C, Quaglino S, et al.: Successful management with 2 overlapping bare stents for post-carotid endarterectomy carotid pseudoaneurysm secondary to carotid shunt. Ann Vasc Surg 29: 594.e1594.e4, 2015

20) Chalouhi N, Starke RM, Koltz MT, et al.: Stent-assisted coiling versus balloon remodeling of wide-neck aneurysms: comparison of angiographic outcomes. AJNR Am J Neuroradiol 34: 1987-1992, 2013

21) Yoon SM, Jo KW, Baik MW, Kim YW: Delayed carotid wallstent shortening resulting in restenosis following successful carotid artery angioplasty and stenting. J Korean Neurosurg Soc 46: 495-497, 2009

22) Walsh KM, Moskowitz SI, Hui FK, Spiotta AM: Multiple overlapping stents as monotherapy in the treatment of 'blister' pseudoaneurysms arising from the supraclinoid internal carotid artery: a single institution series and review of the literature. J Neurointerv Surg 6: 184-194, 2014

23) Fankhauser GT, Stone WM, Fowl RJ, et al.: Surgical and medical management of extracranial carotid artery aneurysms. J Vasc Surg 61: 389-393, 2015

Corresponding author:

Jun Haruma, MD, PhD, Department of Neurosurgery, Fukuyama City Hospital, 5-23-1 Zaocho, Fukuyama, Hiroshima 721-8511, Japan.

$\triangle$ j.haruma619@gmail.com 